\title{
Radial-basis-functions neural network sliding mode control for underactuated mechanical systems
}

\author{
Sonia Mahjoub • Faisal Mnif • Nabil Derbel • \\ Mustapha Hamerlain
}

Received: 14 July 2013 / Revised: 6 February 2014 / Accepted: 19 February 2014 / Published online: 11 March 2014

(C) Springer-Verlag Berlin Heidelberg 2014

\begin{abstract}
This paper presents an indirect adaptive neural network sliding mode Control (IANSMC) technique and a neural network sliding mode control (NNSMC) for underactuated robot manipulators. The adaptive neural network (NN) based on radial basis functions (RBF) is used to estimate the equivalent control and to compensate model uncertainties. In IANSMC, the adaptive learning algorithms are derived using Lyapunov stability analysis. Sliding mode control and indirect adaptive technique are combined to deal with modeling parameter uncertainties and bounded disturbances. The stability of the mixed controller is then proved. NN parameters are tuned on-line, without an off-line learning phase. For the NNSMC, the NN control is used to learn the equivalent control due to the unknown nonlinear system dynamics and the robust sliding mode control (SMC) is designed for a trajectory tracking control. Simulation results show that the NNSMC and IANSMC are better than the classical SMC to control underactuated manipulators. Although the proposed controllers can eliminate the chattering phenomena and estimate matching uncertainties. The IANSMC can also reject mismatched perturbations. Discussions and comparisons between proposed controllers are presented.
\end{abstract}

S. Mahjoub $(\varangle) \cdot$ N. Derbel

Advanced Control and Energy Management Laboratory (CEM-Lab), Sfax Engineering School, University of Sfax, BP W, 3038 Sfax, Tunisia

e-mail: mahjoub_sonia@yahoo.fr

F. Mnif

Department of Electrical and Computer Engineering,

Sultan Qaboos University, Muscat, Oman

M. Hamerlain

Centre for Development of Advanced Technologies, Algiers, Algeria
Keywords Neural networks · Sliding mode control . Lyapunov stability · Indirect adaptive · Underactuated manipulator

\section{Introduction}

Underactuated mechanical systems (UMS) are increasingly present in the field of robotics. They have less actuators than degrees of freedom. In these systems we find: manipulators, vehicles and humanoids with some passive joints. The underactuation arises by deliberate design for the purpose of reducing the weight of the manipulator or might be caused by actuator failure [1].

The difficulty in controlling underactuated mechanisms is due to the fact that techniques developed for fully actuated systems cannot be directly used. These systems are not feedback linearizable, yet they exhibit nonholonomic constraints and nonminimum phase characteristics. Because of this, the class of underactuated mechanical systems present challenging control problems. It is moreover shown that this class of systems can not be stabilized by means of continuous control algorithms. Sliding mode control (SMC) approaches are one of the most common techniques used to control UMS. The effectiveness of the SMC for nonlinear systems in general has been clearly proved by the control engineering community in virtue of its robustness against external perturbations and model parameters variations $[2,3]$. However, in practice such an approach results in a undesirable chattering phenomena, which may excite high-frequency dynamics. Moreover, specifications of the system modeling approximations, as well as the boundaries of model uncertainties and unknown disturbances are usually difficult tasks in most situations. To avoid chattering effects, the boundary layer of the switching surface can be reduced. However this approach does not ensure 
the convergence of the state trajectories of the system on the sliding surface. Moreover, the dynamic analysis within the boundary layer is complicated [4]. On the other hand, the design of sliding-mode controllers needs the knowledge of the mathematical model of the plant, which decreases the performance in some applications where the mathematical modeling of the system is not well defined or when the knowledge of the parameters is incomplete. To overcome this problem, several researchers have used soft computing methodologies like fuzzy logic [5] or artificial neural networks (ANN) [6-9]. The combination between the intelligent control algorithms and the sliding mode approach attracted the attention of many researchers $[8,10,11]$. The idea of combining robust control and NN methods is used as a way to improve the performance and robustness to model imprecisions of the control system.

The purpose of the present study is to design an indirect adaptive neural network sliding mode Control (IANSMC) and a neural network sliding mode control (NNSMC) for underactuated systems. In the first approach, an RBF neural network (NN) control is proposed to compensate the effect of uncertainties and parameter variations with an on-line adaptation of the proposed controller. The adaptive law is employed to on-line adjust the weights of neural, algorithm is derived using a suitable Lyapunov equation. Then the stability of the control system can be guaranteed. In the second approach the NN control is used to learn the equivalent control due to the unknown nonlinear system dynamics and the robust control SMC is designed for trajectory tracking. These proposed controllers are used to control the UMS cartpendulum prototype.

This paper is organized as follows: we firstly introduce in Sect. 2 the system dynamical model. In Sect. 3, we describe the SMC. Then, an IANSMC is proposed later in Sect. 4. In Sect. 5 the NNSMC is presented. Finally in Sect. 6, simulation results are presented and discussed.

\section{System dynamical model}

The cart-pendulum system is a typical benchmark of nonlinear and UMS [12]. For this system, the control input is the force $u$ that moves the cart horizontally, the outputs are the angular position of the pendulum $\theta$ and the linear position of the cart. However, despite its simple mechanical structure, this prototype is not easy to control and requires sufficiently sophisticated control designs. Indeed it is proven that the system is not feedback linearizable and has no constant relative degree [13]. Moreover, Zhao and Spong [14] have shown that many geometric properties of the system are lost when the pendulum moves through horizontal positions. The principal control task considered for the cart pendulum is to swing up the pendulum from the stable equilibrium point to the unstable equilibrium point, and to stabilize the

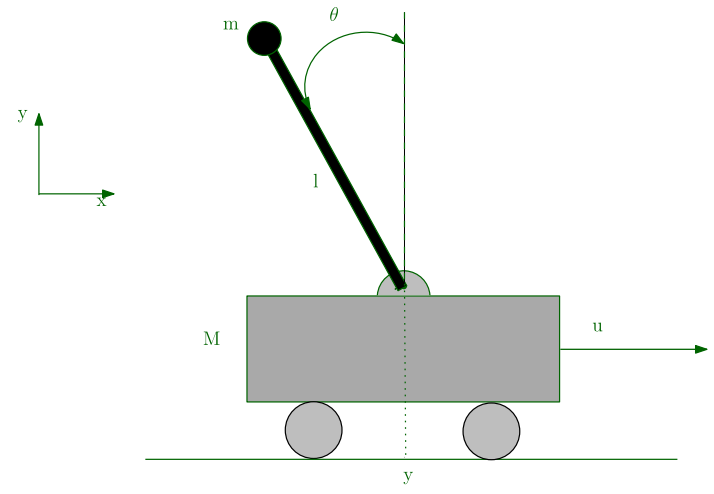

Fig. 1 Cart-pendulum system

cart in a desired position. Numerous control techniques have been developed to stabilize the inverted Pendulum such as; proportional-integral-derivative (PID) controller where the parameters control gains are adjustable and updated online with a stable adaptation mechanism [15]. Fantoni et al. [12] proposed an energy approach. Using this control the system can be stabilized around its homoclinic orbit. Olfati [1] transformed the system in cascade form, then a fixed point backstepping procedures has been developed for global and semi-global stabilization. A hybrid controller has been presented by Spong [14], which globally asymptotically stabilizes the system for all initial conditions. Backstepping procedure [16], SMC [17,18], NN [19,20], fuzzy control [21] are also used to control this system. The dynamical behavior of the inverted pendulum can be described by [12]:

$$
\begin{aligned}
(m+M) \ddot{y}+m L\left(\ddot{\theta} \cos \theta-\dot{\theta}^{2} \sin \theta\right) & =u \\
\ddot{y} \cos \theta+L \ddot{\theta}-g \sin \theta & =0
\end{aligned}
$$

where $L$ is the length of the pendulum, $m$ is the pendulum mass, $M$ is the cart mass, $u$ is the horizontal force action, $\theta$ is the angular deviation, $y$ is the horizontal displacement of the cart (Fig. 1).

Letting $x_{1}=y, x_{2}=\dot{y}, x_{3}=\theta, x_{4}=\dot{\theta}$, we can transform these equation into the following state space canonical representation:

$$
\begin{aligned}
& \dot{x}_{1}=x_{2} \\
& \dot{x}_{2}=f_{1}+b_{1} u \\
& \dot{x}_{3}=x_{4} \\
& \dot{x}_{4}=f_{2}+b_{2} u
\end{aligned}
$$

where $X=\left[x_{1}, x_{2}, x_{3}, x_{4}\right]^{T}$ is the state variable vector and $u$ is the control input. $f_{1}, f_{2}, b_{1}$ and $b_{2}$ are nonlinear functions defined by: 


$$
\begin{aligned}
& f_{1}=\frac{m L x_{4} \sin x_{3}-m g \sin x_{3} \cos x_{3}}{M+m \sin ^{2} x_{3}} ; \\
& f_{2}=\frac{(m+M) g \sin x_{3}-m L x_{4}^{2} \cos x_{3}}{L\left(M+m \sin ^{2} x_{3}\right)} \\
& b_{1}=\frac{1}{M+m \sin ^{2} x_{3}} ; b_{2}=\frac{-\cos x_{3}}{L\left(M+m \sin ^{2} x_{3}\right)}
\end{aligned}
$$

\section{Sliding mode control}

We can transform the system into two subsystems with state variable groups $\left(x_{1}, x_{2}\right)$ and $\left(x_{3}, x_{4}\right)$ for which we construct the following linear functions as sliding surfaces. Those function are defined to be the first level sliding mode and are expressed by

$s_{1}=c_{1} e_{1}+e_{2}$

$s_{2}=c_{2} e_{3}+e_{4}$

$e=\left[\begin{array}{l}e_{1} \\ e_{2} \\ e_{3} \\ e_{4}\end{array}\right]=\left[\begin{array}{l}x_{1}-x_{1 d} \\ x_{2}-x_{2 d} \\ x_{3}-x_{3 d} \\ x_{4}-x_{4 d}\end{array}\right]$

$c_{1}$ and $c_{2}$ are some real positive design parameters and $x_{1 d}, x_{2 d}, x_{3 d}, x_{4 d}$ are the desired values. Differentiating (4) with respect to time and setting $\dot{s}_{1}=0$ and $\dot{s}_{2}=0$ gives the equivalent control laws as:

$u_{e q 1}=-\frac{c_{1} \dot{e_{1}}+f_{1}}{b_{1}} ; \quad u_{e q 2}=-\frac{c_{2} \dot{e_{3}}+f_{2}}{b_{2}}$

The choice of the sliding surface influences to the behavior of the control performance. It can minimize chattering, improving the tracking speed and the error convergence. In this paper a second-level sliding surface $S$ is defined as a linear combination of first level sliding surfaces $s_{1}$ and $s_{2}$. In addition, all system states of are attracted to this surface and the switching control is determined based on stability of $S$ by deriving this surface with respect to time.

For the whole system (2) we define the second-level sliding surface as:

$S=\alpha_{1} s_{1}+\alpha_{2} s_{2}$

where $\alpha_{1}$ and $\alpha_{2}$ are the sliding control parameters.

The control of the system can be written as:

$u=u_{e q}+u_{s w}$

where the equivalent control $u_{e q}$ is designed in such a way that we have $\dot{S} S \leq 0$, hence:

$u_{e q}=\frac{\alpha_{1} b_{1} u_{e q 1}+\alpha_{2} b_{2} u_{e q 2}}{\alpha_{1} b_{1}+\alpha_{2} b_{2}}$

In the following we use the Lyapunov stability theory to find the switching control law $u_{s w}$.
Choosing the Lyapunov condidate function as:

$V=\frac{1}{2} S^{T} S$

the convergence of the sliding mode requires that

$\dot{V}=S \dot{S} \leq-\eta|S|$

where $\eta$ is a positive constant. Computing $\dot{V}$

$$
\begin{aligned}
\dot{V} & =S \dot{S}=S\left(\alpha_{1} \dot{s_{1}}+\alpha_{2} \dot{s_{2}}\right) \\
& =S\left(\alpha_{1}\left(c_{1} \dot{e_{1}}+\dot{e_{2}}\right)+\alpha_{2}\left(c_{2} \dot{e_{3}}+\dot{e_{4}}\right)\right) \\
& =S\left(\alpha_{1}\left(c_{1} \dot{e_{1}}+f_{1}+b_{1} u\right)+\alpha_{2}\left(c_{2} \dot{e_{3}}+f_{2}+b_{2} u\right)\right)
\end{aligned}
$$

Substituting (7), (8) in (11) gives:

$$
\begin{aligned}
\dot{V}= & S\left[\alpha_{1}\left(c_{1} \dot{e_{1}}+f_{1}+b_{1}\left(u_{e q}+u_{s w}\right)\right)\right. \\
& \left.+\alpha_{2}\left(c_{2} \dot{e_{3}}+f_{2}+b_{2}\left(u_{e q}+u_{s w}\right)\right)\right] \\
= & S\left[u_{s w}\left(\alpha_{1} b_{1}+\alpha_{2} b_{2}\right)\right] \leq-\eta|S|
\end{aligned}
$$

then:

$u_{s w}\left(\alpha_{1} b_{1}+\alpha_{2} b_{2}\right) \leq-\eta \operatorname{sign}(S)$

so $u_{s w}$ can be defined by the following term

$u_{s w}=-\frac{\eta \operatorname{sign}(S)}{\alpha_{1} b_{1}+\alpha_{2} b_{2}}$

Consequently the control law (7) becomes

$u=\frac{\alpha_{1} b_{1} u_{e q 1}+\alpha_{2} b_{2} u_{e q 2}}{\alpha_{1} b_{1}+\alpha_{2} b_{2}}-\frac{\eta \operatorname{sign}(S)}{\alpha_{1} b_{1}+\alpha_{2} b_{2}}$

\section{Indirect adaptive technique neural network sliding mode control (IANSMC)}

Consider now the perturbed model of system (2) defined as :

$\dot{x}_{1}=x_{2}$

$\dot{x}_{2}=f_{1}+b_{1} u+d_{1}+\Delta f_{u 1}$

$\dot{x}_{3}=x_{4}$

$\dot{x}_{4}=f_{2}+b_{2} u+d_{2}+\Delta f_{u 2}$

where $d_{1}, d_{2}$ are the matched uncertainties, $\Delta f_{u 1}, \Delta f_{u 2}$ the unmatched uncertainties [11], $f_{1}, f_{2}, b_{1}$ and $b_{2}$ are expressed by:

$f_{1}=f_{10}+\Delta f_{1} ; \quad f_{2}=f_{20}+\Delta f_{2} ;$

$b_{1}=b_{10}+\Delta b_{1} ; \quad b_{2}=b_{20}+\Delta b_{2}$

where $\Delta f_{1}, \Delta f_{2}, \Delta b_{1}, \Delta b_{2}$ denote the uncertain parts, $f_{10}$, $f_{20}, b_{10}, b_{20}$ are nominal values of $f_{1}, f_{2}, b_{1}$ and $b_{2}$ expressed in Eq. (3). 


\subsection{Model description of NN}

Neural networks have the capacity to approximate nonlinear functions and to learn through examples of the system in control engineering [6]. They can be used to approximate the optimal controller which is designed based on the assumption that all dynamics of the system are known. Then a novel adaptive neural controller is developed to compensate the plant uncertainties, smooth the control signal and increase the system robustness. Radial basis function neural networks (RBFNN) have been used in this control structure to compensate the uncertainties in the design of the SMC. An RBFNN has an input layer, a hidden layer and an output layer. Neurons in the hidden layer have gaussian transfer functions for which outputs are inversely proportional to the distance from the center of the neuron. Compared to the standard feedforward backpropagation networks, RBFNNs have the advantage of faster learning speed and less chance of falling into local minimal convergence.

The Gaussian-type function can be expressed as:

$\varphi_{j}(s)=\exp \left[-\frac{\left(s-m_{j}\right)^{2}}{\sigma_{j}^{2}}\right], \quad j=1,2 \ldots k$

$s$, is used as the input signal for a RBF NN, $k$ is the number of nodes in the hidden layer, $m_{j}$ and $\sigma_{j}$ are the center and the variance of the jth basis function. In this section, the IANSMC algorithm for nonlinear underactuated systems is described, the adapting rule is derived, and the stability analysis is discussed. In the indirect case, two approximations are used to estimate the dynamics of the controlled system, which will be used in the control law [8]. An arbitrary choice of the initial value of adjustable parameters cannot always ensure the convergence of the adaptive algorithm due to the singularity problem. Differentiating (9) with respect to time, yields:

$$
\begin{aligned}
\dot{V}= & S\left[\alpha_{1}\left(c_{1} e_{2}+f_{10}+\Delta f_{1}+\left(b_{10}+\Delta b_{1}\right) u+d_{1}+\Delta f_{u 1}\right)\right. \\
& \left.+\alpha_{2}\left(c_{2} e_{4}+f_{20}+\Delta f_{2}+\left(b_{2}+\Delta b_{20}\right) u+d_{2}+\Delta f_{u 2}\right)\right] \\
= & S\left[\alpha_{1}\left(c_{1} e_{2}+f_{10}+b_{10} u\right)\right. \\
& \left.+\alpha_{2}\left(c_{2} e_{4}+f_{20}+b_{20} u\right)+\Psi\right]
\end{aligned}
$$

where

$$
\begin{aligned}
\Psi= & \alpha_{1}\left(\Delta f_{1}+\Delta b_{1} u+d_{1}+\Delta f_{u 1}\right) \\
& +\alpha_{2}\left(\Delta f_{2}+\Delta b_{2} u+d_{2}+\Delta f_{u 2}\right)
\end{aligned}
$$

An RBF NN will be presented to estimate and compensate the effect of uncertainties and $\bar{\Psi}$ is the output of NN estimating $\Psi$ [20].

The proposed control $u$ is taken as:

$$
u=\frac{\alpha_{1} b_{10} u_{e q 1}+\alpha_{2} b_{20} u_{e q 2}}{\alpha_{1} b_{1}+\alpha_{2} b_{2}}-\frac{\bar{\Psi}+\eta \operatorname{sign}(S)}{\alpha_{1} b_{1}+\alpha_{2} b_{2}}
$$

where $\eta$ is a positive constant.

Moreover, an adaptive adjusting law for parameters will be designed. As a second approach, an estimation of system parameters can be done.

The output of the linear layer RBF NN is:

$\bar{\Psi}=\bar{\Psi}(s, m, \sigma)=\sum_{j=1}^{k} W_{j} \varphi_{j}(s)=W^{T} \varphi(s)$

where:

$W=\left[\begin{array}{c}W_{1} \\ W_{2} \\ . . \\ W_{k}\end{array}\right] ; \varphi(s)=\left[\begin{array}{c}\varphi_{1}(s) \\ \varphi_{2}(s) \\ . . \\ \varphi_{k}(s)\end{array}\right] ; m=\left[\begin{array}{c}m_{1} \\ m_{2} \\ . . \\ m_{k}\end{array}\right] ; \sigma=\left[\begin{array}{c}\sigma_{1} \\ \sigma_{2} \\ . . \\ \sigma_{k}\end{array}\right]$

The sliding variable $s$ is an input signal to the RBF neural network, $W$ is the output weight vector of the RBFNN.

An optimal NN controller will be designed to estimate the uncertainties (17) such that:

$\Psi=\hat{\Psi}+\varepsilon_{1}=\hat{W}^{T} \hat{\varphi}(s)+\varepsilon_{1}$

where $\varepsilon_{1}$ is a reconstructed error vector. $\hat{\Psi}$ is an optimal output of NN, function $\hat{W}$ and $\hat{\varphi}$ corresponds to optimal parameters of the NN.

The expression of the control law becomes:

$u=u_{e q}+u_{s w}+u_{N N}$

where

$$
\begin{aligned}
u_{N N} & =\frac{-\bar{\Psi}}{\alpha_{1} b_{1}+\alpha_{2} b_{2}} ; \quad u_{s w}=\frac{-\eta \operatorname{sign}(S)}{\alpha_{1} b_{1}+\alpha_{2} b_{2}} ; \\
u_{e q} & =\frac{\alpha_{1} b_{1} u_{e q 1}+\alpha_{2} b_{2} u_{e q 2}}{\alpha_{1} b_{1}+\alpha_{2} b_{2}}
\end{aligned}
$$

with $\bar{\Psi} \in \mathbb{R}$. Substituting (21) in (16) gives:

$$
\begin{aligned}
\dot{V} & =S[\Psi-\bar{\Psi}-\eta \operatorname{sign}(S)] \\
& =-\eta|S|+o(\Delta W, \Delta m, \Delta \sigma)
\end{aligned}
$$

where $\Delta W=\tilde{W}=\hat{W}-W, \Delta m=\tilde{m}=\hat{m}-m, \Delta \sigma=$ $\tilde{\sigma}=\hat{\sigma}-\sigma$

\subsection{Adaptation law}

In this part, a learning algorithm for tuning parameters $W, m$ and $\sigma$ is given. The linearization technique is used to transform the nonlinear basis function into a partially linear form of the active function [9]. Differentiating (19) yields:

$$
\begin{aligned}
\Delta \bar{\Psi}_{j}(s)= & \Delta W_{j} \varphi_{j}(s)+W_{j} \frac{\partial \varphi_{j}}{\partial m_{j}} \Delta m_{j} \\
& +W_{j} \frac{\partial \varphi_{j}}{\partial \sigma_{j}} \Delta \sigma_{j}+\varepsilon_{2 j}
\end{aligned}
$$

where $\bar{\Psi}_{j}(s)=W_{j} \Psi_{j}(s)$ and $\bar{\Psi}(s)=\sum_{j=1}^{k} \bar{\Psi}_{j}(s)$. Then $\Delta \bar{\Psi}(s)=\sum_{j=1}^{k} \Delta \bar{\Psi}_{j}(s)$ and $\varepsilon_{2}=\sum_{j=1}^{k} \varepsilon_{2 j} \in \mathbb{R}$ denotes 
the error vector due to the neglection of high-order terms of the Taylor expansion of $\bar{\Psi}(s)$ with a maximum bound given by $\left|\varepsilon_{2}\right| \leq \varepsilon_{2 \max }$.

where $\frac{\partial \varphi_{j}}{\partial m_{j}}$ and $\frac{\partial \varphi_{j}}{\partial \sigma_{j}}$ are given by:

$\begin{aligned} & \varphi_{m}^{\prime}=\varphi_{m}^{\prime T}= {\left[\begin{array}{ccccc}\frac{\partial \varphi_{1}}{\partial m_{1}} & 0 & 0 & . . & 0 \\ 0 & \frac{\partial \varphi_{2}}{\partial m_{2}} & 0 & . . & 0 \\ . . & . . & . . & . . & . \\ . . & . . & . . & . . & \frac{\partial \varphi_{k}}{\partial m_{k}}\end{array}\right] \in \mathbb{R}^{k \times k} } \\ & \varphi_{\sigma}^{\prime}=\varphi_{\sigma}^{\prime T}=\left[\begin{array}{ccccc}\frac{\partial \varphi_{1}}{\partial \sigma_{1}} & 0 & 0 & . . & 0 \\ 0 & \frac{\partial \varphi_{2}}{\partial \sigma_{2}} & 0 & . . & 0 \\ . . & . . & . . & . \\ . . & . . & . . & . & \frac{\partial \varphi_{k}}{\partial \sigma_{k}}\end{array}\right] \in \mathbb{R}^{k \times k}\end{aligned}$

The difference between the system uncertainties and the $\mathrm{NN}$ output is expressed as:

$\tilde{\Psi}=\Psi-\bar{\Psi}=\hat{\Psi}+\varepsilon_{1}-\bar{\Psi}$

Then (26) can be expressed as:

$\tilde{\Psi}=\Delta \bar{\Psi}+\varepsilon_{1}$

with $\Delta \bar{\Psi}=\hat{\Psi}-\bar{\Psi}$.

Equations (27) and (24) give:

$\tilde{\Psi}(s)=\tilde{W}^{T} \varphi(s)+W^{T} \varphi_{m}^{\prime}(\tilde{m})+W^{T} \varphi_{\sigma}^{\prime}(\tilde{\sigma})+\varepsilon_{1}+\varepsilon_{2}$

where the uncertain term $\varepsilon_{1}+\varepsilon_{2}$ is assumed to be bounded by $2\left|\varepsilon_{1}+\varepsilon_{2}\right|<\eta$. The adaption laws of the NN controller are designed using the following equations:

$$
\left\{\begin{array} { l } 
{ \dot { \tilde { W } } = - \dot { W } = \eta _ { 1 } S \varphi ( s ) } \\
{ \dot { \tilde { m } } = - \dot { m } = \eta _ { 2 } S \varphi _ { m } ^ { \prime } W } \\
{ \dot { \tilde { \sigma } } = - \dot { \sigma } = \eta _ { 3 } S \varphi _ { \sigma } ^ { \prime } W }
\end{array} \Rightarrow \left\{\begin{array}{l}
\dot{\tilde{W}}_{j}=-\dot{W}_{j}=\eta_{1} S \varphi_{j}(s) \\
\dot{\tilde{m}}_{j}=-\dot{m}_{j}=\eta_{2} S \frac{\partial \varphi_{j}}{\partial m_{j}} W_{j} \\
\dot{\tilde{\sigma}}_{j}=-\dot{\sigma}_{j}=\eta_{3} S \frac{\partial \varphi_{j}}{\partial \sigma_{j}} W_{j}
\end{array}\right.\right.
$$

where $\eta_{i}$ are are some positive constants, $i=\{1,2,3\}$ and for $j=\{1, . ., k\}$.

\subsection{Stability proof}

Consider the Lyapunov function candidate:

$V=\frac{1}{2} S^{2}+\frac{1}{2 \eta_{1}} \tilde{W}^{T} \tilde{W}+\frac{1}{2 \eta_{2}} \tilde{m}^{T} \tilde{m}+\frac{1}{2 \eta_{3}} \tilde{\sigma}^{T} \tilde{\sigma}$

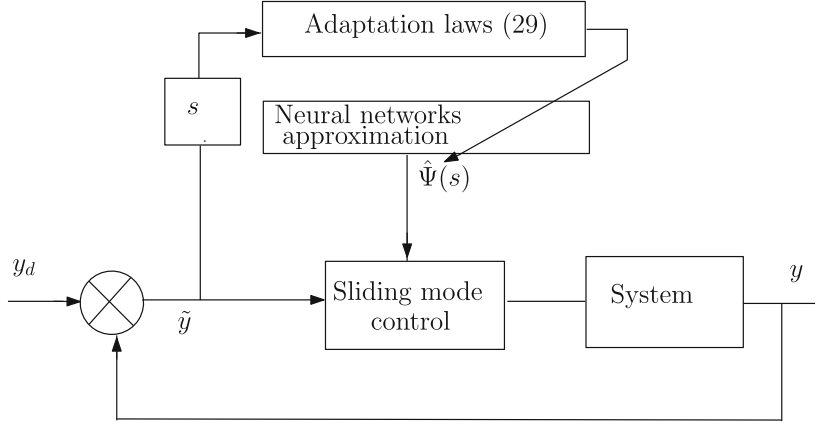

Fig. 2 Structure of IANSMC

Differentiating (30) with respect to time, leads to:

$$
\begin{aligned}
\dot{V}= & S \dot{S}+\frac{1}{\eta_{1}} \tilde{W}^{T} \dot{\tilde{W}}+\frac{1}{\eta_{2}} \tilde{m}^{T} \dot{\tilde{m}}+\frac{1}{\eta_{3}} \tilde{\sigma}^{T} \dot{\tilde{\sigma}} \\
= & -\eta|S|+S \tilde{\Psi}+\frac{1}{\eta_{1}} \tilde{W}^{T} \dot{\tilde{W}}+\frac{1}{\eta_{2}} \tilde{m}^{T} \dot{\tilde{m}}+\frac{1}{\eta_{3}} \tilde{\sigma}^{T} \dot{\tilde{\sigma}} \\
= & -\eta|S|+S\left[\tilde{W}^{T} \varphi(s)+W^{T} \varphi_{m}^{\prime}(\tilde{m})+W^{T} \varphi_{\sigma}^{\prime}(\tilde{\sigma})\right. \\
& \left.+\varepsilon_{1}+\varepsilon_{2}\right]-\frac{1}{\eta_{1}} \tilde{W}^{T} \dot{W}-\frac{1}{\eta_{2}} \tilde{m}^{T} \dot{m}-\frac{1}{\eta_{3}} \tilde{\sigma}^{T} \dot{\sigma} \\
= & \tilde{W}^{T}\left(\varphi(s) S-\frac{1}{\eta_{1}} \dot{W}\right)+\tilde{m}^{T}\left(\varphi_{m}^{\prime}(\tilde{m}) W S-\frac{1}{\eta_{2}} \dot{m}\right) \\
& +\tilde{\sigma}^{T}\left(\varphi_{\sigma}^{\prime}(\tilde{\sigma}) W S-\frac{1}{\eta_{3}} \dot{\sigma}\right)-\eta|S|+2 S\left(\varepsilon_{1}+\varepsilon_{2}\right) \\
= & -\eta|S|+2 S\left(\varepsilon_{1}+\varepsilon_{2}\right) \leq-\eta|S|+2\left|\varepsilon_{1}+\varepsilon_{2}\right||S| \\
= & -\left(\eta-2\left|\varepsilon_{1}+\varepsilon_{2}\right|\right)|S| \leq 0
\end{aligned}
$$

$\dot{V}$ becomes negative if $2\left|\varepsilon_{1}+\varepsilon_{2}\right|<\eta$. Thus the IANSMC control system is stable.

In summary, an indirect RBF SMC law is developed in (21) with parameters $W, m$ and $\sigma$ adjusted by (29). The objective is to build a RBF NN control in order to compensate the effect of uncertainties and the parameter variations, with an on-line adaptation of the proposed controller. The adaptive NN SMC is stable in the sense of Lyapunov. The representation of the proposed IANSMC is shown in Fig. 2.

\section{NN sliding mode controller (NNSMC)}

\subsection{Control principle}

If the knowledge of $f$ and $b$ is very poor, then the computed equivalent control will be too far from the actual equivalent control. The model used is an estimate of the real system. To solve this problem, a NN is proposed to compute the equivalent control to control the underactuated manipulator. A RBFNN is employed to model the relation between the system state vector, $x$, and the equivalent control, $u_{e q}$. The model of the con- 


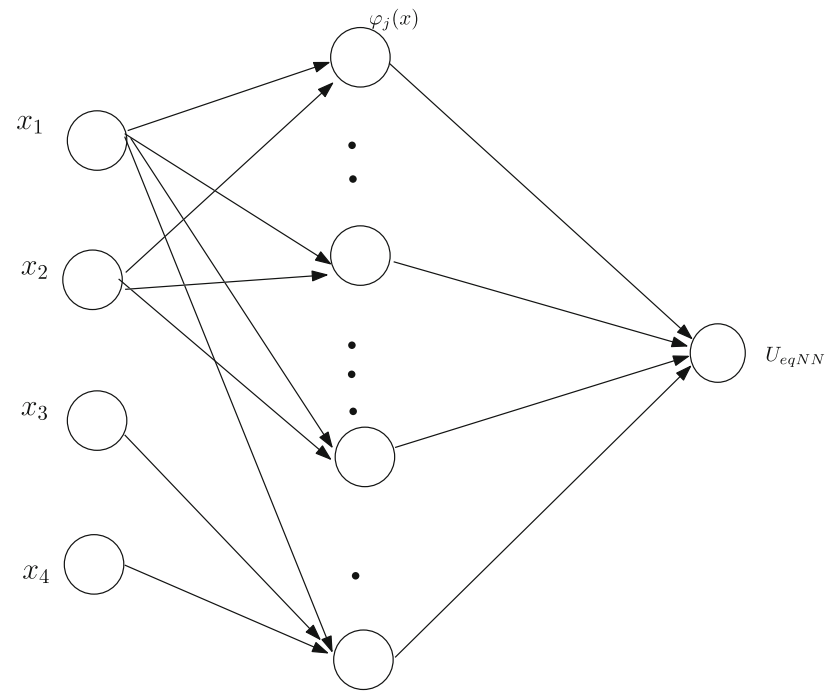

Fig. 3 Disposition of neurons

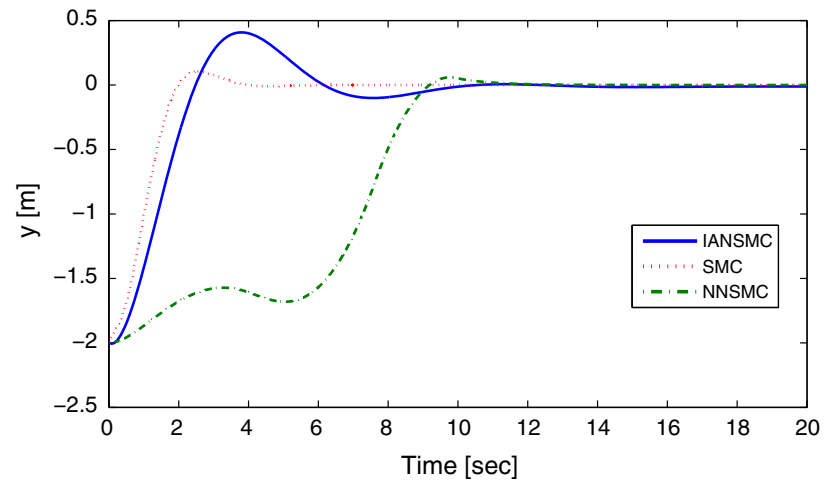

Fig. 4 Evolution of the position error $y$ without perturbation

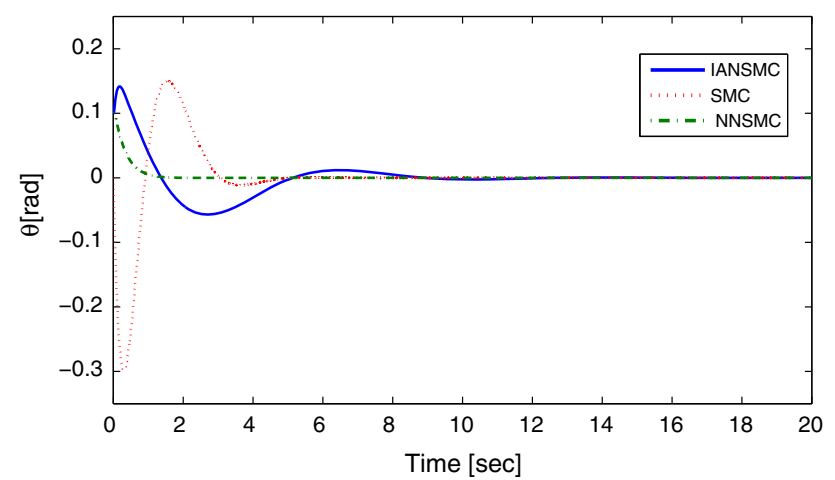

Fig. 5 Evolution of the angle error $\theta$ without perturbation

trol is presented in Fig. 3. The Gaussian function is used as the activation function of each neuron in the hidden layer.

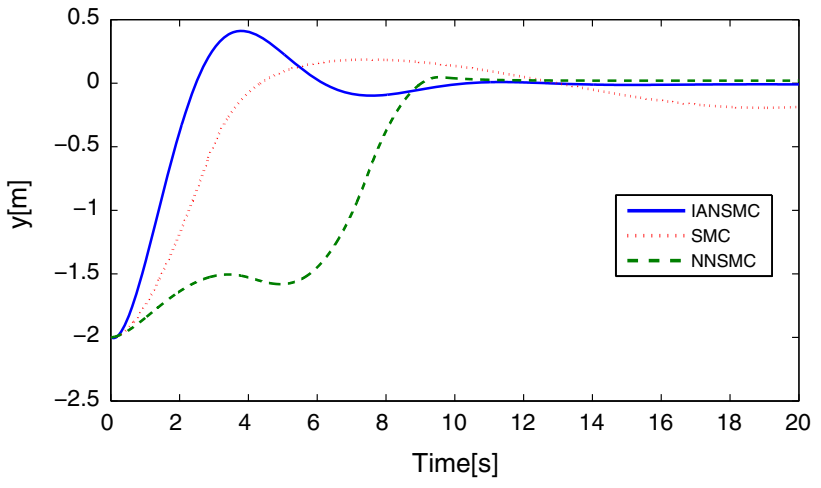

Fig. 6 Evolution of the position error $y$ with perturbations

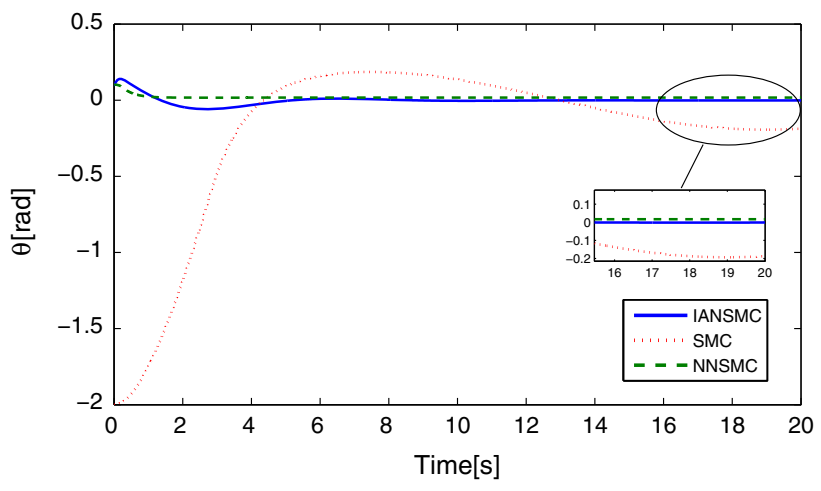

Fig. 7 Evolution of the angle error $\theta$ with perturbations

\subsection{NN sliding mode controller}

Sliding surfaces are defined as (4) for system (2). The secondlevel sliding surface is defined by (6) and the equivalent SMC can be chosen as:

$U_{e q}=k_{11} \dot{e_{1}}+k_{12} \dot{e_{2}}+k_{21} x_{2}+k_{22}$

where:

$k_{11}=\frac{-\alpha_{1} c_{1} l\left(M+m \sin ^{2} x_{3}\right)}{\alpha_{1} l-\alpha_{2} \cos x_{3}} ;$

$k_{12}=\frac{-\alpha_{2} c_{2} l\left(M+m \sin ^{2} x_{3}\right)}{\alpha_{1} l-\alpha_{2} \cos x_{3}}$

$k_{21}=\frac{\alpha_{1} m l^{2} \sin x_{3}+\alpha_{2} m g}{\alpha_{1} l-\alpha_{2} \cos x_{3}} ;$

$k_{22}=\frac{\alpha_{1} m l g \sin x_{3} \cos x_{3}-\alpha_{2}(M+m) g \sin x_{3}}{\alpha_{1} l-\alpha_{2} \cos x_{3}}$

In this case the switching control is defined by:

$u_{s w}=-K \operatorname{sign}(S)$

Using the neural control, the expression of $U_{e q}$ becomes:

$U_{e q}=U_{e q N N}+o(\Delta f, \Delta b)$

where $\Delta f=\Delta f_{1}+\Delta f_{2}$ and $\Delta b=\Delta b_{1}+\Delta b_{2}$. 


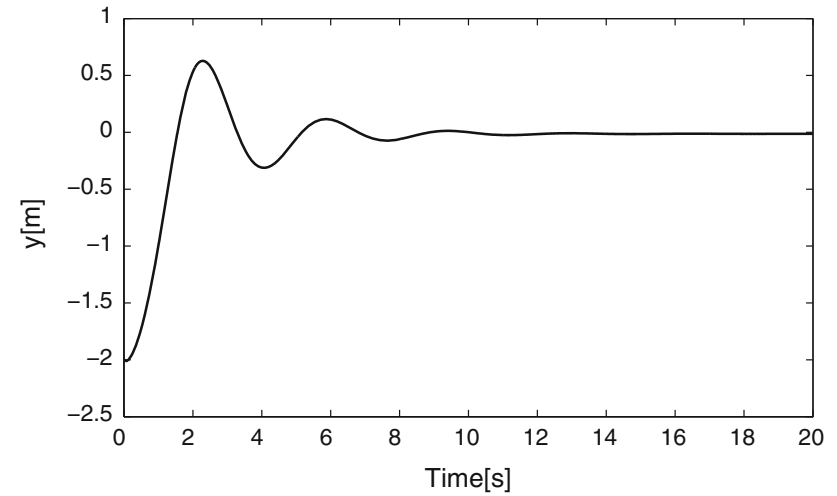

Fig. 8 Evolution of the position error $y$ with unmatched and matched perturbations (IANSMC)

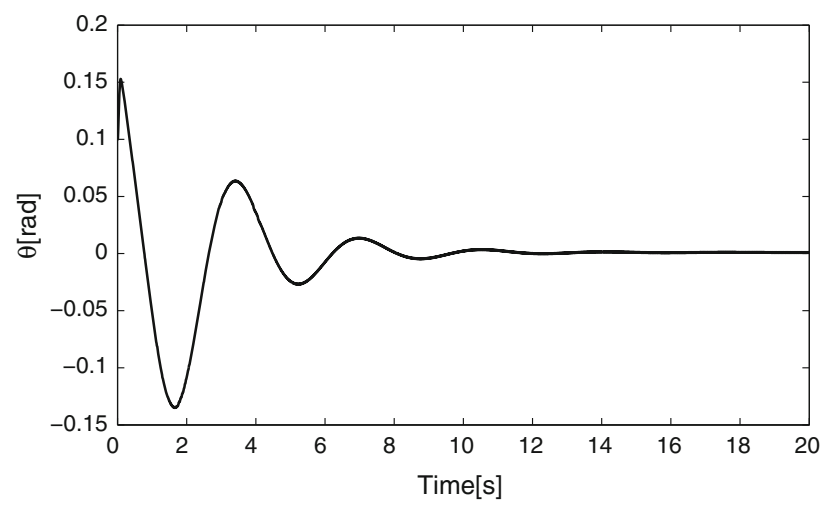

Fig. 9 Evolution of the angle error $\theta$ with unmatched and matched perturbations (IANSMC)

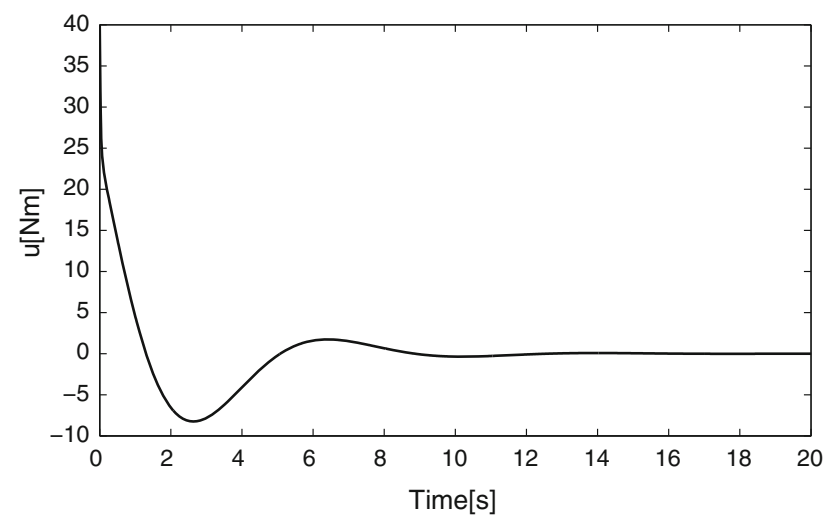

Fig. 10 The simulated torque $u$ without perturbation (SMC) using saturation function

The total control is expressed by:

$u=U_{e q N N}+u_{s w}$

Taking into account the Lyapunov function in (7), we have:

$$
\begin{aligned}
\dot{V} & =S\left(b_{1} \alpha_{1}+b_{2} \alpha_{2}\right)(-K \operatorname{sign}(S)+o(\Delta f, \Delta b)) \\
& =-K|S|\left(b_{1} \alpha_{1}+b_{2} \alpha_{2}\right)+o(\Delta f, \Delta b)
\end{aligned}
$$

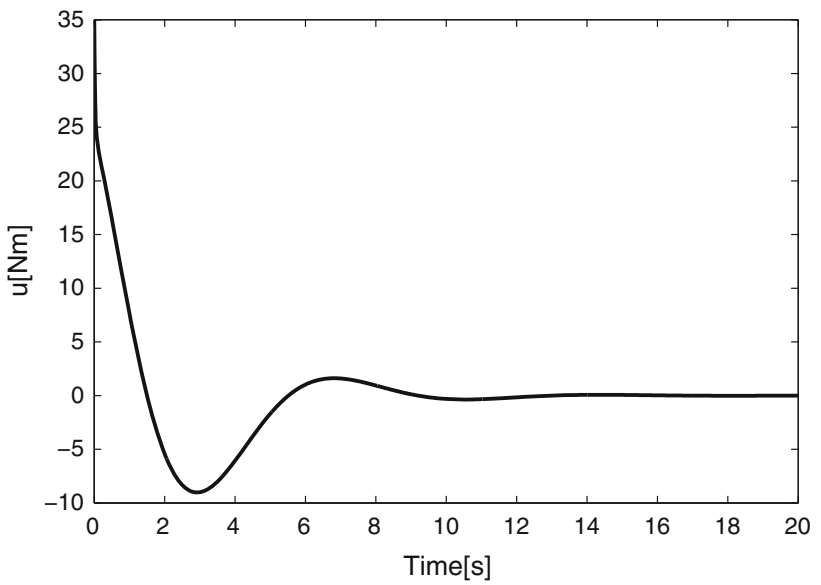

Fig. 11 The simulated torque $u$ without perturbation (IANSMC)

Based on Lyapunov stability theorem, if $V>0$ and $\dot{V} \leq 0$, the stability of the system can be proved. Let $K>0$ and to guarantee that $\dot{V}$ is negative $-K|S|\left(b_{1} \alpha_{1}+\right.$ $b_{2} \alpha_{2}$ ) should be $\leq 0$, so $\alpha_{1}>-\frac{b_{2} \alpha_{2}}{b_{1}}$ then $\alpha_{1}>$ $\alpha_{2} \frac{\cos x_{3}}{l^{2}}$. For the simulations, we choose $\alpha_{1}=2 / l^{2}$ and $\alpha_{2}=1$.

\section{Simulation results and discussion}

The effectiveness of the proposed approaches is evaluated on a 2 degree of freedom underactuated manipulator. For the simulation the following parameters are considered: $M_{0}=$ $20 \mathrm{~kg}, m_{0}=1.8 \mathrm{~kg}, L_{0}=0.3 \mathrm{~m}$.

The initial conditions are considered to be $\left(y_{0}, \dot{y_{0}}\right)=$ $(0,0),\left(\theta_{0}, \dot{\theta_{0}}\right)=(0.1,0)$ and the expectations are $y_{d}=$ 2, $\theta_{d}=0$ and $\dot{y_{d}}=\dot{\theta_{d}}=0$.

Figures 4 and 5 show the simulation results for each of the three controllers (SMC, IANSMC, NNSMC) when $20 \%$ of mass uncertainty is considered for the pendulum and cart in the dynamics of the system and without perturbations. We can note that the convergence is obtained for all the system states. Figures 6, 7, 12 and 14 show the system response when matched disturbances are considered $\left(d_{1}=0.2\right.$ and $d_{2}=$ $0.7 \sin \frac{\pi t}{12}$ ). Unlike the SMC technique, the two controllers IANSMC and NNSMC are robust against these disturbances with slight advantage to the IANSMC.

Figures 8 and 9 present the simulations results for the IANSMC technique when the system is subjected to matched and an unmatched uncertainly defined by: $\Delta f_{u 1}(x)=$ $0.22 x_{1} \cos x_{1}$ and $\Delta f_{u 2}(x)=1.2 x_{3} \cos x_{2}$. All the considered disturbances are rejected by the designed controller.

As shown in Fig. 10 the phenomena of chattering was solved using a function saturation in SMC. Figures 11, 12 and 13 show the simulation results of the control effort for 


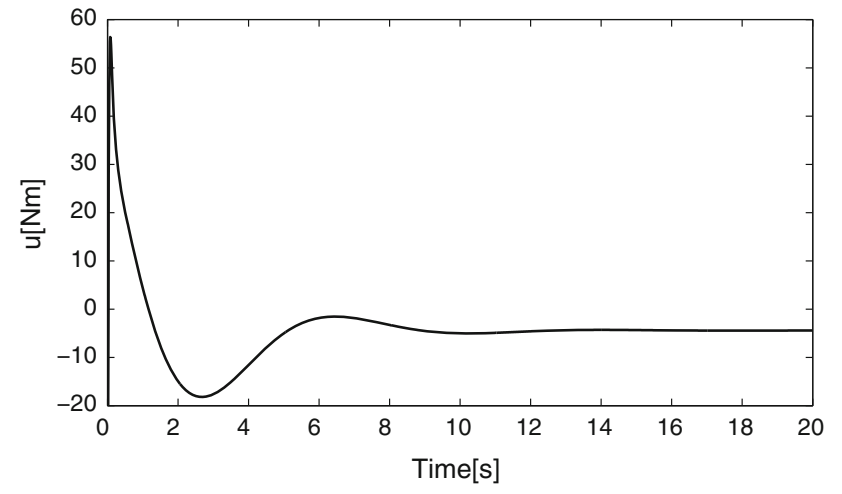

Fig. 12 The simulated torque $u$ with perturbation (IANSMC)

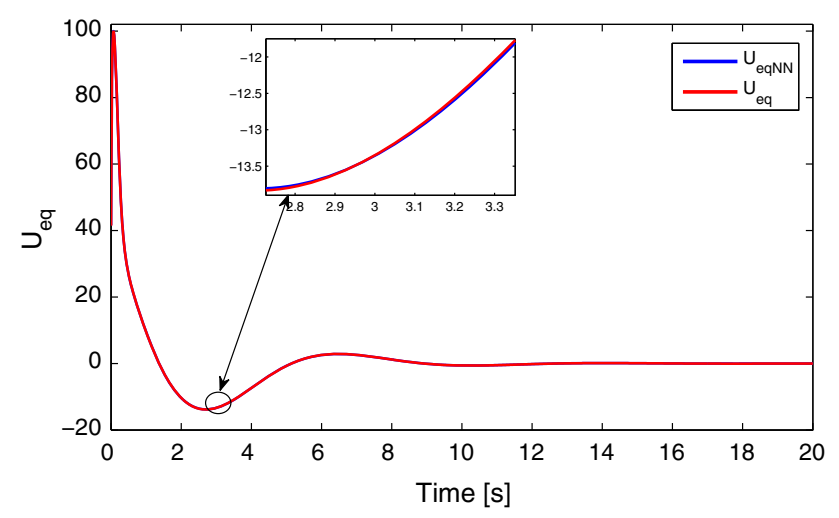

Fig. 13 Learning the equivalent control

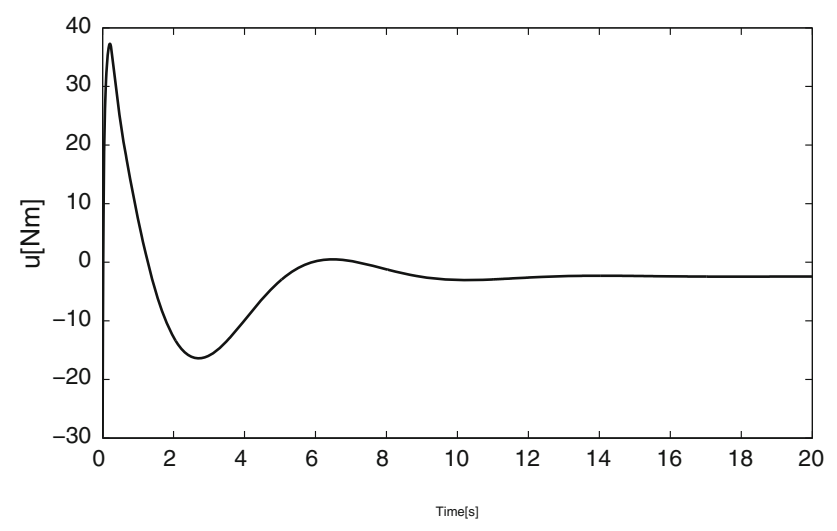

Fig. 14 The simulated torque $u$ NNSMC with perturbation

both the IANSMC and the NNSMC. It is clear that no chattering phenomenon is present. We remark from Fig. 14 a good learning of equivalent control $u_{e q}$ determined from NN. The curves $u_{e q}$ and $u_{e q N N}$ are almost identical.

\section{Conclusion}

In this paper, an IANSMC and NNSMC control techniques have been designed for underactuated manipulators. An RBF
$\mathrm{NN}$ is used to approximate the unknown uncertainties part. An adaptive learning algorithm is constructed based on Lyapunov stability analysis where the weights of the output layer of RBF and the parameters of the basis function are updated on-line. The proposed controllers guarantee stability and convergence of the system position. Simulations have been carried out to evaluate the performance of the proposed controllers. Simulation results show that the proposed IANSMC is able to effectively compensate the parameter uncertainties of the underactuated manipulator better than NNSMC approach and the SMC.

\section{References}

1. Olfati S (2001) Nonlinear control of underactuated mechanical systems with application to robotics and aerospace vehicles. Ph.D. dissertation, Massachusetts Institute of Technology, Cambridge

2. Zhou K, Doyle JC (1998) Essentials of robust control. PrenticeHall, California

3. Liu J, Wang X (2011) Advanced sliding mode control for mechanical systems. Tsinghua University, Beijing and Springer, Berlin Heidelberg

4. Peng J, Wang Y, Sun W, Liu Y (2006) A neural network sliding mode controller with application to robotic manipulator. In: Intelligent Control and Automation, WCICA 2006. The Sixth World Congress on, vol 1, pp 2101-2105. IEEE, 2006

5. Liu H, Zhang T (2012) Fuzzy sliding mode control of robotic manipulators with kinematic and dynamic uncertainties. J Dyn Syst Meas Control 134(6):061007

6. Sun T, Pei H, Pan Y, Zhou H, Zhang C (2011) Neural network-based sliding mode adaptive control for robot manipulators. Neurocomputing 74:2377-2384

7. Liu H, Zhang T (2012) Adaptive neural network finite-time control for uncertain robotic manipulators. J Intell Robot Syst 134(6):1-15

8. Baruch L (2007) Direct and indirect adaptive neural control of nonlinear systems. Stud Fuzziness Soft Comput 208:95-114

9. Wai RJ (2003) Tracking control based on neural network strategy for robot manipulator. Neurocomputing 5:425-445

10. Hung LC, Chung HY (2007) Decoupled control using neural network-based sliding-mode controller for nonlinear systems. Expert Syst Appl 32:1168-1182

11. Wang SW, Yu DW, Yu DL (2011) Compensation for unmatched uncertainty with adaptive RBF network. Int J Eng Sci Tech 3(6):3543

12. Fantoni I, Lozano R (2002) Non-linear control for underactuated mechanical systems. Springer, London

13. Jakubczyk B, Respondek W (1980) On the linearization of control systems. Bult Acad Polon Sei Math 28:517-522

14. Zhao J, Spong MW (2001) Hybrid control for global stabilization of the cart pendulum system. Automatica 37(12):1941-1951

15. Chang WD, Hwang RC, Hsieh JG (2002) A self-tuning PID control for a class of nonlinear systems based on the Lyapunov approach. J Process Control 12:233-242

16. Elsayed BA, Hassan MA, Mekhilef S (2013) Decoupled third-order fuzzy sliding model control for cart-inverted pendulum system. Appl Math Info Sci 7(1):193-201

17. Lewis AS (2007) Nonsmooth optimization and robust control. Annu Rev Control 31:167-177

18. Mahjoub S, Mnif F, Derbel N (2011) Set point stabilization of a 2DOF underactuated manipulator. J comput 6(2):368-376 
19. Anderson CW (1989) Learning to control an inverted pendulum using neural networks. IEEE Control Syst Mag 9:31-37

20. Mahjoub S, Mnif F, Derbel N (2013) Radial-basis-functions neural network sliding mode control for underactuated manipulators. In: IEEE International Multi-Conference on Systems, Signals and Devices (SSD), Hammamet, Tunisie

21. Yi J, Yubazaki N, Hirota K (2001) Upswing and stabilization control of inverted pendulum system based on the SIRMs dynamically connected fuzzy inference model. Fuzzy Set Syst 122(1):139-152 